The Astrophysical Journal, 619:L47-L50, 2005 January 20

(c) 2005. The American Astronomical Society. All rights reserved. Printed in U.S.A.

\title{
THE GALEX-VVDS MEASUREMENT OF THE EVOLUTION OF THE FAR-ULTRAVIOLET LUMINOSITY DENSITY AND THE COSMIC STAR FORMATION RATE
}

D. Schiminovich, ${ }^{1,2}$ O. Ilbert ${ }^{3}$ S. Arnouts, ${ }^{3}$ B. Milliard,${ }^{3}$ L. Tresse, ${ }^{3}$ O. Le Fèvre, ${ }^{3}$ M. Treyer, ${ }^{2,3}$ T. K. Wyder, ${ }^{2}$ T. Budavári, ${ }^{4}$ E. Zucca,${ }^{5}$ G. Zamorani, ${ }^{5}$ D. C. Martin, ${ }^{2}$ C. Adami, ${ }^{3}$ M. Arnaboldi, ${ }^{6}$ S. Bardelli, ${ }^{5}$ T. Barlow, ${ }^{2}$ L. Bianchi, ${ }^{4}$ M. Bolzonella, ${ }^{7}$ D. Bottini, ${ }^{8}$ Y.-I. Byun,${ }^{9}$ A. Cappi,${ }^{5}$ T. Contini, ${ }^{10}$ S. Charlot,${ }^{11,12}$ J. Donas, ${ }^{3}$ K. Forster, ${ }^{2}$ S. Foucaud,${ }^{10}$ P. Franzetti,${ }^{10}$ P. G. Friedman, ${ }^{2}$ B. Garilli, ${ }^{10}$ I. Gavignaud, ${ }^{10}$ L. Guzzo, ${ }^{13}$ T. M. Heckman, ${ }^{4}$ C. Hoopes, ${ }^{4}$ A. Iovino, ${ }^{13}$ P. Jelinsky, ${ }^{14}$ V. Le Brun, ${ }^{3}$ Y.-W. Lee, ${ }^{9}$ D. Maccagni, ${ }^{10}$ B. F. Madore, ${ }^{15}$ R. Malina, ${ }^{3}$ B. Marano, ${ }^{7}$ C. Marinoni, ${ }^{3}$ H. J. McCracken, ${ }^{12}$ A. Mazure, ${ }^{3}$ B. Meneux,${ }^{3}$ P. Morrissey, ${ }^{3}$ S. Neff,${ }^{16}$ S. Paltani, ${ }^{3}$ R. Pellò, ${ }^{10}$ J. P. Picat,${ }^{10}$ A. Pollo, ${ }^{13}$ L. Pozzetti,${ }^{5}$ M. Radovich, ${ }^{6}$ R. M. Rich, ${ }^{17}$ R. Scaramella,${ }^{8}$ M. Scodeggio, ${ }^{10}$ M. Seibert ${ }^{3}$ O. Siegmund, ${ }^{14}$ T. Small, ${ }^{2}$ A. S. Szalay, ${ }^{4}$ G. Vettolani, ${ }^{18}$ B. Welsh, ${ }^{15}$ C. K. Xu, ${ }^{19}$ And A. Zanichelli ${ }^{18}$ Received 2004 June 30; accepted 2004 October 28; published 2005 January 17

\section{ABSTRACT}

In a companion Letter (Arnouts et al.) we present new measurements of the galaxy luminosity function at $1500 \AA$ out to $z \sim 1$ using Galaxy Evolution Explorer VIMOS-VLT Deep Survey observations (1039 galaxies with NUV $\leq 24.5$ and $z>0.2$ ) and at higher $z$ using existing data sets. In this Letter we use the same sample to study evolution of the FUV luminosity density $\rho_{1500}$. We detect evolution consistent with a $(1+z)^{2.5 \pm 0.7}$ rise to $z \sim 1$ and $(1+z)^{0.5 \pm 0.4}$ for $z>1$. The luminosity density from the most UV-luminous galaxies (UVLGs) is undergoing dramatic evolution (30 times) between $0<z<1$. UVLGs are responsible for a significant fraction $(>25 \%)$ of the total far-ultraviolet luminosity density at $z \sim 1$. We measure dust attenuation and star formation rates of our sample galaxies and determine the star formation rate density $\left(\dot{\rho}_{\star}\right)$ as a function of redshift, both uncorrected and corrected for dust. We find good agreement with other measures of $\dot{\rho}_{\star}$ in the rest ultraviolet and $\mathrm{H} \alpha$ given the still significant uncertainties in the attenuation correction.

Subject headings: cosmology: observations - galaxies: evolution galaxies: luminosity function, mass function — ultraviolet: galaxies

\section{INTRODUCTION}

The rest-frame far-ultraviolet (FUV; $1500 \AA$ ) luminosity has been used to determine the star formation rate (SFR) of stellar populations over the complete range of redshifts for which

\footnotetext{
${ }^{1}$ Department of Astronomy, Columbia University, MC 2457, 550 West 120th Street, New York, NY 10027.

${ }^{2}$ California Institute of Technology, MC 405-47, 1200 East California Boulevard, Pasadena, CA 91125.

${ }^{3}$ Laboratoire d'Astrophysique de Marseille, BP 8, Traverse du Siphon, 13376 Marseille Cedex 12, France.

${ }^{4}$ Department of Physics and Astronomy, Johns Hopkins University, Homewood Campus, Baltimore, MD 21218.

${ }^{5}$ Osservatorio Astronomico di Bologna, via Ranzani, I-40127 Bologna, Italy.

${ }^{6}$ Osservatorio Astronomico di Capodimonte, via Moiariello 16, I-80131 Naples, Italy.

${ }^{7}$ Departimento di Astronomia, Università di Bologna, via Ranzani 1, I40127 Bologna, Italy.

${ }^{8}$ IASF-INAF, Sezione di Milano, Via E. Bassini 15, I-20133 Milan, Italy.

${ }^{9}$ Center for Space Astrophysics, Yonsei University, Seoul 120-749, Korea.

${ }^{10}$ Laboratoire d'Astrophysique, Observatoire Midi-Pyrénées, 14, Avenue Edouard Belin, Toulouse F-31400, France.

${ }^{11}$ Max Planck Institut fur Astrophysik, Karl-Schwarzschild-Strasse 1, Postfach 1317, D-85741 Garching, Germany.

${ }^{12}$ Institut d'Astrophysique de Paris, UMR 7095, 98 bis Boulevard Arago, 75014 Paris, France.

${ }^{13}$ Osservatorio Astronomico di Brera, via Brera 28, I-20121 Milan, Italy.

${ }^{14}$ Space Sciences Laboratory, University of California at Berkeley, 601 Campbell Hall, Berkeley, CA 94720.

${ }^{15}$ Observatories of the Carnegie Institution of Washington, 813 Santa Barbara Street, Pasadena, CA 91101.

${ }^{16}$ Laboratory for Astronomy and Solar Physics, NASA Goddard Space Flight Center, Greenbelt, MD 20771.

${ }^{17}$ Department of Physics and Astronomy, University of California at Los Angeles, Box 951547, Knudsen Hall, Los Angeles, CA 90095.

${ }^{18}$ Istituto di Radioastronomia, CNR, via Gobetti 101, I-40129 Bologna, Italy.

${ }^{19}$ NASA/IPAC, California Institute of Technology, MC 100-22, 770 South Wilson Avenue, Pasadena, CA 91125.
}

galaxies have been observed. The utility and limitations of the integrated measures - the FUV luminosity function $\left(\phi_{\mathrm{FUV}}\right)$ and luminosity density $\left(\rho_{1500}\right)$ - and their relation to the star formation history of the universe have been extensively discussed and reviewed (e.g., Madau et al. 1998; Hopkins 2004). A principal goal of the Galaxy Evolution Explorer (GALEX) mission (Martin et al. 2005) is to perform deep wide-angle surveys to obtain an accurate measurement of the evolution of the FUV luminosity density (LD) over the range $0<z<1$ and beyond. In this Letter we present results from a small pilot study performed in the $2 \mathrm{hr}$ VIMOS-VLT Deep Survey (VVDS) field using measurements from 1039 galaxies.

GALEX data will allow us to determine how the rest-UV can best be used to study the detailed properties of galaxies (e.g., dust, metallicity, and star formation history). Since this is work in progress, here we instead use existing methods to determine the intrinsic luminosity of galaxies in the FUV (Meurer et al. 1999, hereafter M99, for dust corrections) and the SFR that this luminosity implies (Kennicutt 1998 for SFR conversion). This simple analysis yields some quick answers; we discuss how this work will be expanded and developed in the near future.

Throughout this Letter we adopt the flat $\lambda$ cosmology $\left(\Omega_{M}=0.3, \Omega_{\Lambda}=0.7\right)$ with $H_{0}=70 \mathrm{~km} \mathrm{~s}^{-1} \mathrm{Mpc}^{-1}$.

\section{DATA}

GALEX observations of the VVDS 0226-04 field $\left(02^{\mathrm{h}} 26^{\mathrm{m}} 00^{\mathrm{s}},-04^{\circ} 30^{\prime} 00^{\prime \prime}, \mathrm{J} 2000.0\right)$ were performed in 2004 October-November as part of the GALEX Deep Imaging Survey. Further details of these observations, the subsequent match to VVDS spectroscopy and photometry, and the calculation of the luminosity function (LF) can be found in the companion 
TABLE 1

FUV 1500 Å Luminosity Density

\begin{tabular}{|c|c|c|c|}
\hline \multirow[b]{2}{*}{$\langle z\rangle$} & \multicolumn{3}{|c|}{$\log \rho_{1500}{ }^{a}$} \\
\hline & Total & $V_{\max }$ & $L>L_{\min }{ }^{\mathrm{b}}$ \\
\hline $0.055_{-0.055}^{+0.045 c}$ & $25.54_{-0.02}^{+0.09}$ & & 23.97 \\
\hline $0.3 \pm 0.1 \ldots \ldots \ldots$ & $25.86_{-0.05}^{+0.05}$ & $25.86_{-0.05}^{+0.05}$ & $24.67_{-0.18}^{+0.17}$ \\
\hline $0.5 \pm 0.1 \ldots \ldots \ldots$ & $25.97_{-0.08}^{+0.15}$ & $25.76_{-0.05}^{+0.05}$ & $25.20_{-0.09}^{+0.09}$ \\
\hline $0.7 \pm 0.1 \ldots$ & $26.16_{-0.13}^{+0.31}$ & $25.91_{-0.05}^{+0.05}$ & $25.48_{-0.05}^{+0.05}$ \\
\hline $1.0 \pm 0.2 \ldots$ & $26.11_{-0.13}^{+0.31 d}$ & $25.69_{-0.05}^{+0.05}$ & $25.51_{-0.05}^{+0.05}$ \\
\hline $2.0 \pm 0.5 \ldots \ldots \ldots$ & $26.45_{-0.09}^{+0.25}$ & $26.30_{-0.04}^{+0.04}$ & $26.03_{-0.12}^{+0.13}$ \\
\hline $2.9 \pm 0.5 \ldots \ldots \ldots$ & $26.52_{-0.07}^{+0.17}$ & $26.40_{-0.03}^{+0.03}$ & $26.26_{-0.08}^{+0.08}$ \\
\hline $3.0 \pm 0.24^{\mathrm{e}} \ldots \ldots$ & $25.58_{-0.17}^{+0.31}$ & $\ldots$ & 26.22 \\
\hline
\end{tabular}

${ }^{\text {a }}$ In units of ergs s${ }^{-1} \mathrm{~Hz}^{-1} \mathrm{Mpc}^{-3}$, flat $\lambda$ cosmology with

$H_{0}=70 \mathrm{~km} \mathrm{~s}^{-1} \mathrm{Mpc}^{-1}\left(\Omega_{M}=0.3, \Omega_{\Lambda}=0.7\right)$.

${ }^{\mathrm{b}} L_{\min }=0.2 L_{\star}(z=3)^{d} ; M_{\min }=-19.32$.

${ }^{\mathrm{c}}$ Data from Wyder et al. (2005).

${ }^{\mathrm{d}}$ Fixed faint-end slope $\alpha=-1.6$.

${ }^{\mathrm{e}}$ Data from Steidel et al. (1999).

Letter Arnouts et al. (2005, hereafter Paper I) and references therein. Paper I also describes the derivation of the $\phi_{\mathrm{Fuv}}$ at $z=2.0$ and 2.9 using a Hubble Deep Field (HDF) sample from Arnouts et al. (2002). For comparison we also use the local $\phi_{\mathrm{Fuv}}$ (Wyder et al. 2005) and the $z \sim 3$ Lyman break galaxy (LBG) $\phi_{\mathrm{Fuv}}$ (Steidel et al. 1999)

\section{LUMINOSITY DENSITY}

We calculated the FUV LD $\rho_{1500}(z)$ from the GALEX-VVDS sample in four redshift bins $(\langle z\rangle=0.3,0.5,0.7,1.0)$ and also determined values for the HDF sample $(\langle z\rangle=2.0,2.9)$. Results are shown in Table 1 and plotted in Figure 1. We chose to calculate $\rho_{1500}(z)$ in several ways. First we summed $\phi(L) L d L$ using the LF obtained from the $V_{\max }$ method. Because luminosity bins with no detections do not contribute, we consider this a lower limit on $\rho_{1500}(z)$. We also calculated a "total" LD by integrating Schechter function fits to the LF using the formula

$$
\rho=\int_{L_{\min }}^{\infty} d L L \phi(L)
$$

with $L_{\min }=0\left[\rho=\phi_{*} L_{*} \Gamma(\alpha+2)\right]$. Although this quantity is strongly dependent on uncertainties in the faint-end slope $(\alpha)$, it allows direct comparison with other measurements of $\rho_{1500}(z)$ and the SFR density, $\dot{\rho}_{\star}$. Fits and errors were determined using the ALF tool (Ilbert et al. 2004) with error bars based on the extreme values of the $\mathrm{LD}$ calculated at each point on the $\alpha-M_{\star} 1 \sigma$ error contour. For the $z=1.0$ bin, our best Schechter function fit yielded large errrors for the slope $\left(\alpha=-1.63_{-0.43}^{+0.45}\right)$. For this bin we fixed the faint-end slope at $\alpha$ to -1.6 , adopting the value used in high- $z$ studies (e.g., Steidel et al. 1999) and consistent within errors with our own values at lower and higher $z$. Total $\rho_{1500}(z)$ shows significant $\sim(1+z)^{2.5}$ evolution out to $z \sim 1$, with evidence for a shallow continued rise out to $z=3$. This evolution is discussed further in the next section. Two points are worth noting regarding the comparison of LD at different redshifts. First, as demonstrated in Paper I and discussed below, the galaxy population that contributes most of the LD varies (vs. color and luminosity) with redshift. Second, while most of the sample is UV-selected, the Steidel et al. (1999) LBG galaxies were color-selected, and the $z=3 \mathrm{LD}$ value may be missing some fraction of the UV light. The similarity between the $z=2.9$ and 3 data points suggests that the missing fraction is small.

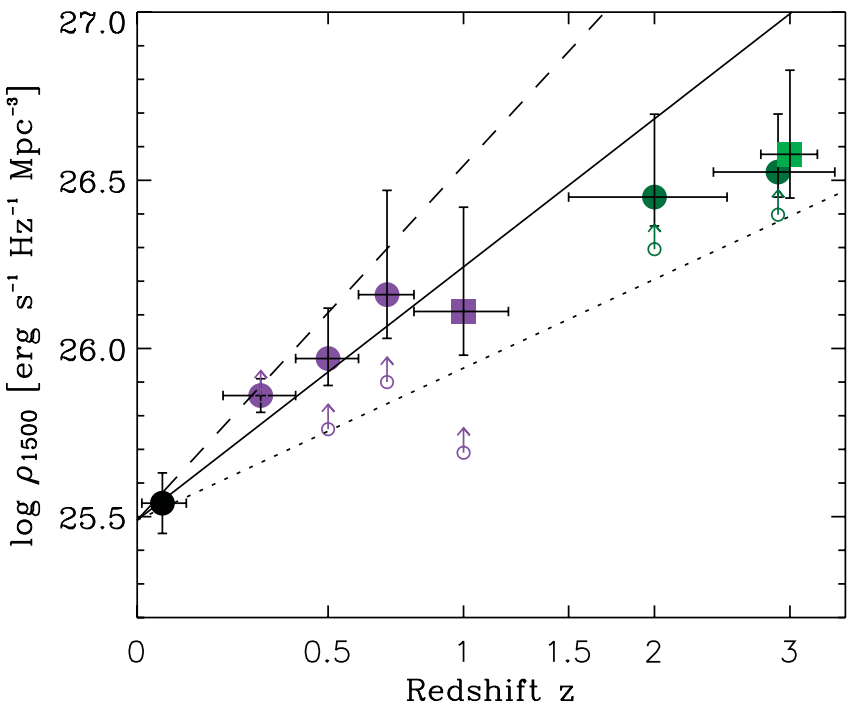

FIG. 1.-FUV LD vs. redshift. Filled circles indicate LF fit to full sample with unconstrained slope $\alpha$. Filled squares denote LF fit with fixed $\alpha=-1$.6. Purple $(G A L E X)$ and dark green (HDF) symbols are from this work. Black filled circle (bottom left) is taken from local LF (Wyder et al. 2005) and green square from Steidel et al. (1999). Open circles denote $\rho_{1500}$ determined using $V_{\max }$. Errors do not include cosmic variance. Lines indicate $(1+z)^{n}$ evolution. Dotted, solid, and dashed lines correspond to $n=1.5,2.5$, and 3.5 , respectively.

We explore the contribution to the LD from UV-luminous galaxies (UVLGs) by measuring the LD from galaxies with $L>L_{\min }$. To facilitate comparison with high- $z$ studies, we set $L_{\min }=0.2 L_{\star, z=3}\left(M_{\min }=-19.32\right)$ from Steidel et al. (1999), also adopted by Giavalisco et al. (2004) for their work. ${ }^{20}$ These galaxies are observable in all redshift ranges, and therefore there is no additional uncertainty related to extrapolation beyond the faintest observed magnitude. Figure 2 highlights the dramatic evolution of $\rho_{1500 \text {, UVLG }}$, increasing by 30 times to

\footnotetext{
${ }^{20}$ This luminosity corresponds to $10^{10.1} L_{\odot}, \sim_{\frac{2}{3}}$ the luminosity limit $\left(10^{10.3} L_{\odot}\right)$ adopted for UVLGs in Heckman et al. (2005).
}

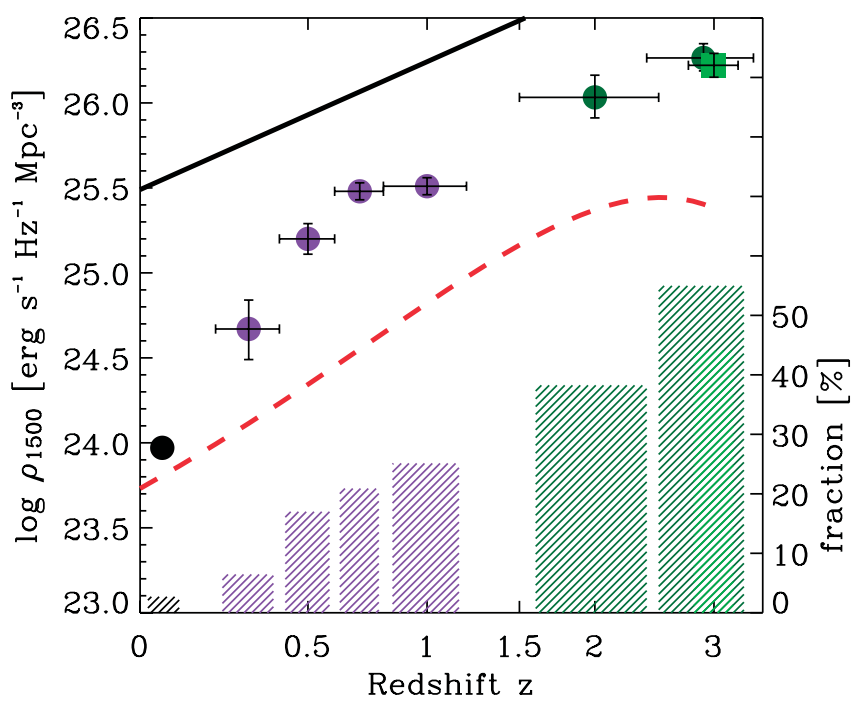

FIG. 2.-FUV LD of UVLGs vs. redshift and comparison with QSO LD. Filled circles from $\rho_{1500 \text {, UvLG }}$ integrated from $\infty$ down to $0.2 L_{\star, z=3}$ or $M_{\mathrm{FUV}}-$ 19.32. Colors same as in Fig. 1. Vertical hatched bars indicate fraction of luminosity emitted by galaxies brighter than $0.2 L_{\star, z=3}$. Red dashed line shows QSO FUV LD using values from Boyle et al. (2000) and Madau et al. (1999). Solid line is the same as in Fig. 1. 


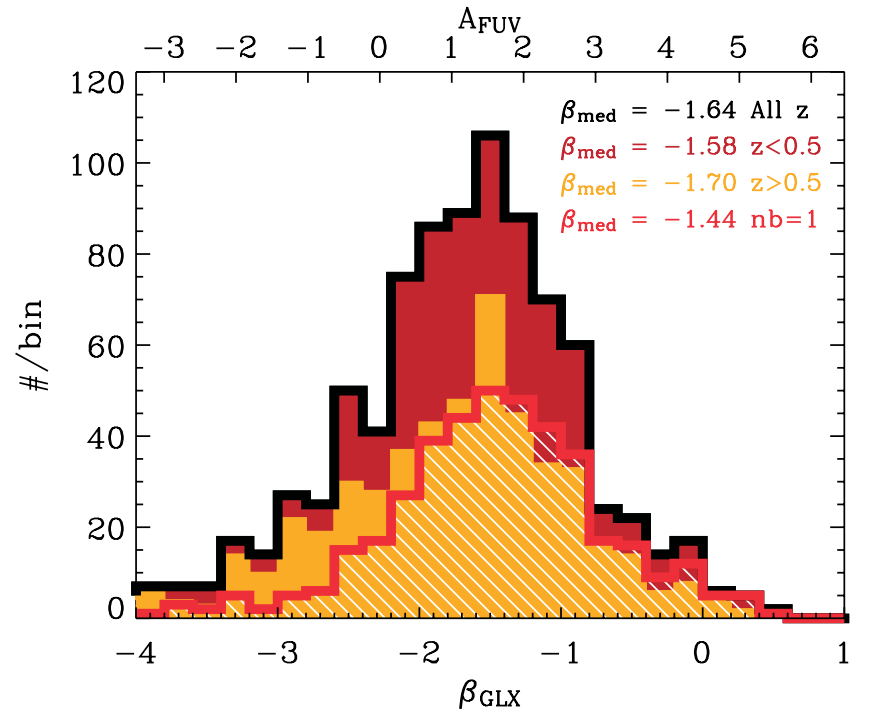

FIG. 3.-Histogram of FUV slope $\beta_{G L X}$ for 888 galaxies with measurements in $U$ band (black) and split into two subsamples: $z<0.5$ (brown) and $z>$ 0.5 (orange). Red/white: Distribution of $\beta_{G L X}$ for "isolated" GALEX detections with only one optical counterpart within 4 " radius.

$z \sim 1$ or $(1+z)^{5}$. Furthermore, we find that UVLGs are a major contributor to $\rho_{1500}$ at $z \sim 1$ with a fractional contribution, $\rho_{1500 \text {, UVLG }} / \rho_{1500}$, of $25 \%$. We plot for comparison $\rho_{1500 \text {, QSO }}$ using the functional form of the QSO LD evolution (in the $B$ band) from Boyle et al. (2000) and the QSO spectral energy distribution from Madau et al. (1999), which has a shallower evolutionary slope (3.5) versus UVLGs (5) for $z<1$.

\section{STAR FORMATION RATE DENSITY}

To determine intrinsic ultraviolet luminosities for the GALEXVVDS sample, we apply the M99 dust attenuation formula

$$
A_{\mathrm{FUV}}=4.43+1.99(\beta)=4.49+1.97\left(\beta_{G L X}\right) \text {, }
$$

where we use the definition of $\beta_{G L X}$, the FUV slope calculated using the rest-frame GALEX FUV and NUV bands, from Kong et al. (2004). We only calculate $\beta_{G L X}$ for the subset of galaxies observed in the $U$ band (888 galaxies). Typical errors are $\sigma_{\beta} \sim$ 0.4 . Figure 3 shows the distribution of the $k$-corrected $\beta_{G L X}$. The full sample has median $\beta_{G L X}=-1.64, \operatorname{FWHM}(\beta)=1.4$ with little variation with redshift. We find good agreement with measurements of $\beta$ at low $-z(\langle\beta\rangle=-1.6$ for an FUV-selected sample; Treyer et al. 2005) and high- $z$ (Adelberger \& Steidel 2000).

Within our own sample we might have expected to see an increase of $\beta_{G L X}$ versus $z$ since high-luminosity galaxieswhich dominate the high- $z$ bins - are expected to show significant attenuation. Several effects could work against this trend. We are detecting galaxies close to the NUV-band confusion limit (beam/source $\sim 10$ for NUV $<25$ ), and source blending could shift UV-optical colors and the slope blueward. We performed tests that conservatively apportioned NUV flux among all potential optical counterparts and set a limit on the offset of the median $\Delta \beta_{G L X, \text { blend }} \leq 0.35$. This is consistent with the median $\beta_{G L X}=-1.44$ measured for "isolated" UV detections with only a single optical counterpart (see Fig. 3). (However, we cannot neglect the possibility that some fraction with multiple counterparts are physical pairs that could show a different distribution of $\left.\beta_{G L X}\right)$. We also note that the M99 $A_{\mathrm{FUV}}-\beta$ relation was determined for starbursting galaxies (the bulk of our sam-

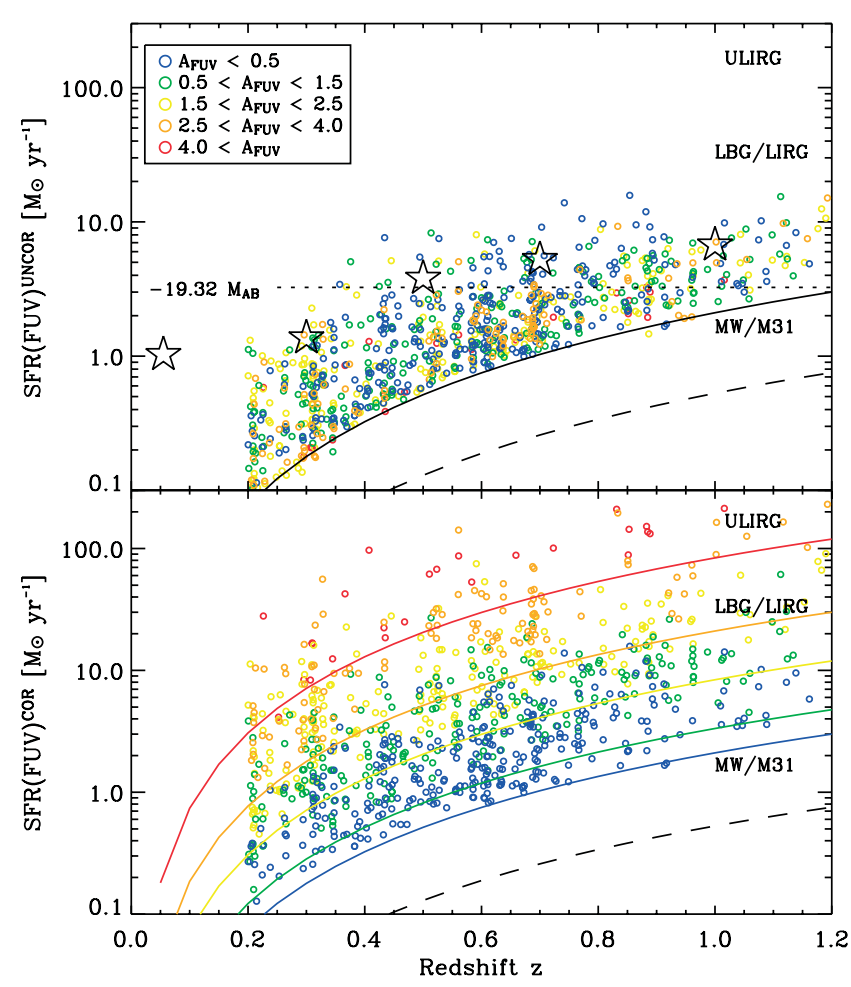

FIG. 4.-SFR of GALEX-VVDS galaxies vs. redshift using Kennicutt (1998) conversion. Top: Uncorrected SFR for galaxies with varying $A_{\mathrm{FUV}}$. Solid line and dashed line correspond to NUV $<24.5$ (current sample) and NUV $<26$ (GALEX Ultra-Deep Survey) limits. Stars show values for $L_{\star}$ from Paper I and Wyder et al. (2005). Dotted line corresponds to $L_{\min }=0.2 L_{\star, z=3}$ cut. Bottom: Attenuation-corrected SFRs. Symbols same as top panel. Colored lines correspond to detection limits for NUV $<24.5$ at minimum attenuation level for each subsample.

ple; see Paper I) but might overestimate the correction for normal star-forming galaxies (Bell 2002; Kong et al. 2004) that are found in our lowest redshift bins. For a conservative measurement of the average attenuation in our whole sample, we use the isolated subsample and calculate a mean attenuation factor of times $7\left(A_{\mathrm{FUV}}^{\mathrm{meas}}=1.8\right)$, where we have estimated and applied a bias correction to the mean (times 0.7 ) because of nonnegligible $\sigma_{\beta}$. We also adopt a "minimum attenuation" $A_{\mathrm{FUV}}^{\min }=1$, which may be more representative of a full UVselected population (Buat et al. 2005).

The SFR was calculated for each galaxy using

$$
\operatorname{SFR}\left(M_{\odot} \mathrm{yr}^{-1}\right)=1.4 \times 10^{-28} L_{\mathrm{FUV}}\left(\operatorname{ergs~s}^{-1} \mathrm{~Hz}^{-1}\right)
$$

from Kennicutt (1998). In Figure 4 we plot the SFR derived for each galaxy using the uncorrected and the dust-corrected FUV luminosities. Our sample shows no dependence of dust attenuation with SFR ${ }^{\text {uncor }}$, and as a consequence we find higher attenuation in galaxies with high SFR ${ }^{\text {cor }}$. This paucity of lowattenuation galaxies with high $\mathrm{SFR}^{\mathrm{cor}}$ has been noted in previous studies (e.g., Wang \& Heckman 1996; Adelberger \& Steidel 2000). Some of the observed effect may also be due to the scatter in $A_{\mathrm{FUV}}$ discussed above (resulting in a tail of high $A_{\mathrm{FUV}}$ galaxies) and/or limitations of the dust attenuation law. We plot $\dot{\rho}_{\star}(z)$ (derived from $\rho_{1500}$ with no dust correction) in Figure 5. Measurements from this Letter were fitted using the parameterization from Baldry et al. (2002) $\left[\dot{\rho}_{\star}(z) \sim(1+z)^{\beta_{\text {evol }}, z<1}\right.$ and $\left.\dot{\rho}_{\star}(z) \sim(1+z)^{\alpha_{\text {evol }}}, z>1\right]$. We find a best-fit $\beta_{\text {evol }}=$ $2.5 \pm 0.7, \alpha_{\text {evol }}=0.5 \pm 0.4$. The $1 \sigma$ constraint on the $\left(\alpha_{\text {evol }}\right.$, 


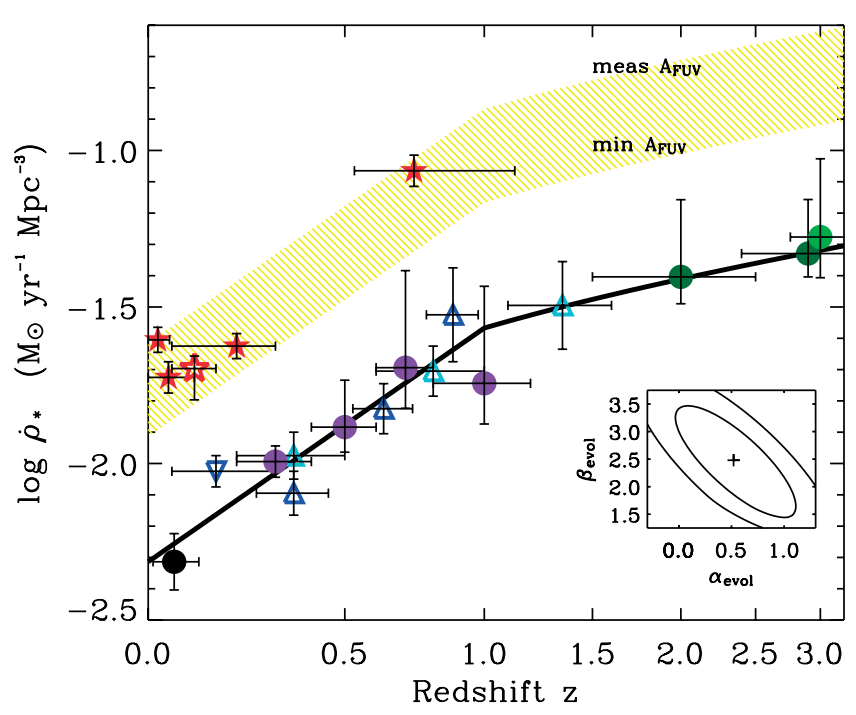

FIG. 5.-SFR density vs. $z$. Filled circles from measurements at $1500 \AA$ (uncorrected for dust) same as in Fig. 1. Blue comparison points are rest-frame UV measurements uncorrected for dust attenuation. Inverted blue triangle from Sullivan et al. (2000). Dark blue triangles from Lilly et al. (1996). Light blue triangles from Wilson et al. (2002) for $\alpha=-1.5$. Solid line rises as $(1+$ $z)^{2.5}$ for $z<1$ and then $(1+z)^{0.5}$ for $z>1$ based on $\chi^{2}$ fit to our sample (see inset; $1 \sigma$ and $2 \sigma$ confidence contours shown). Shaded region shows range corresponding to maximum/minimum dust attenuation. Filled red stars from dust-corrected $\mathrm{H} \alpha$ measurements (with increasing redshift) from PérezGonzález et al. (2003), Gronwall (1999), Tresse \& Maddox (1998), and Tresse et al. (2002). Open red star from SDSS (H $\alpha /$ emission line; Brinchmann et al. 2004).

$\beta_{\text {evol }}$ ) pair is consistent with independent derivations using the Two-Degree Field (Baldry et al. 2002), the Sloan Digital Sky Survey (SDSS; Brinchmann et al. 2003), and other recent studies (e.g., Fig. 13 in Baldry et al. 2002).

Several uncorrected (blue) and dust-corrected (red) comparison measurements obtained using spectroscopic redshifts are shown in Figure 5. Before determining $\dot{\rho}_{\star}$, we converted $\rho_{2000}$ (Sullivan et al. 2000; Lilly et al. 1996) and $\rho_{2500}$ (Wilson et al. 2002, $\alpha=-1.5$ data) to $\rho_{1500}$ using $\rho(\lambda)$ obtained from local $\rho_{1540}$ and $\rho_{2300}$ by Wyder et al. $2005\left(\sim \lambda^{0.9}\right)$. Wilson et al.
(2002) and Lilly et al. (1996) both show good agreement with our measured values despite the difference in evolutionary slope obtained in the two studies $\left(\beta_{\text {evol }} \sim 1.7 \pm 1,3.3 \pm 0.7\right.$, respectively). The local LD reported by Sullivan et al. (2000) appears high, as noted in Wyder et al. (2005). Finally, we show a likely range of dust-corrected SFR densities, applying the average $A_{\mathrm{FUV}}^{\mathrm{min}}, A_{\mathrm{FUV}}^{\text {meas }}$ to the best-fit parameterized $\dot{\rho}_{\star}(z)$. Using the Kennicutt (1998) SFR conversion, we find that recent dustcorrected $\mathrm{H} \alpha$ measurements fall within our attenuationcorrected range. Although we have implicitly assumed no evolution in the dust correction, we emphasize that for UV flux-limited samples we might expect evolution in the average dust-attenuation correction versus redshift, and we will explore this further in future work.

The FUV is tracing a predominantly homogeneous population (star-forming and starbursting), making interpretation of integrated measures much more straightforward than at longer wavelengths (cf. Wolf et al. 2003). We have shown that a significant population of UVLGs lies within easy reach $(0.6<z<1.2)$. We will compare these unique star-forming galaxies with their high-redshift LBG analogs (e.g., Shapley et al. 2003). In the near future our sample will expand by 5 times in this field alone and by more than 100 times using data from redshift surveys across the sky. In some locations we will increase our depth to $m_{\mathrm{AB}} \sim 26$ as part of the Ultra-Deep Imaging Survey and probe down to $0.1 L_{\star}$ (see Fig. 4) to better constrain the faint end of $\phi_{\mathrm{Fuv}}$. This will be supplemented by an even larger catalog (more than $10^{6}$ objects) with photometric redshifts. We will soon be able to determine how SFR evolution depends on environment, morphology, and spectral type and will examine our results within the context of cosmological simulations. A major challenge lies in understanding the role of dust obscuration, one that we will explore using recent, more sophisticated models (e.g., Kong et al. 2004) as the GALEX surveys continue.

GALEX is a NASA Small Explorer, launched in 2003 April. We gratefully acknowledge NASA's support for construction, operation, and science analysis for the GALEX mission, developed in cooperation with the Centre National d'Etudes Spatiales of France and the Korean Ministry of Science and Technology.

\section{REFERENCES}

Adelberger, K. L., \& Steidel, C. C. 2000, ApJ, 544, 218

Arnouts, S., et al. 2002, MNRAS, 329, 355

-. 2005, ApJ, 619, L43

Baldry, I. K., et al. 2002, ApJ, 569, 582

Bell, E. 2002, ApJ, 577, 150

Boyle, B. J., Shanks, T., Croom, S. M., Smith, R. J., Miller, L., Loaring, N., \& Heymans, C. 2000, MNRAS, 317, 1014

Brinchmann, J., Charlot, S., White, S. D. M., Tremonti, C., Kauffmann, G., Heckman, T., \& Brinkmann, J. 2004, MNRAS, 351, 1151

Buat, V., et al. 2005, ApJ, 619, L51

Giavalisco, M., et al. 2004, ApJ, 600, L103

Gronwall, C. 1999, in AIP Conf. Proc. 470, After the Dark Ages: When

Galaxies Were Young, ed. S. Holt \& E. Smith (New York: AIP), 335

Heckman, T. M., et al. 2005, ApJ, 619, L35

Hopkins, A. M. 2004, ApJ, 615, 209

Ilbert, O., et al. 2004, MNRAS, 351, 541

Kennicutt, R. C., Jr. 1998, ARA\&A, 36, 189

Kong, X., Charlot, S., Brinchmann, J., \& Fall, S. M. 2004, MNRAS, 349, 769

Lilly, S. J., Le Fèvre, O., Hammer, F., \& Crampton, D. 1996, ApJ, 460, L1

Madau, P., Haardt, F., \& Rees, M. 1999, ApJ, 514, 648
Madau, P., Pozzetti, L., \& Dickinson, M. 1998, ApJ, 498, 106

Martin, D. C., et al. 2005, ApJ, 619, L1

Meurer, G. R., Heckman, T. M., \& Calzetti, D. 1999, ApJ, 521, 64 (M99)

Pérez-González, P. G., Zamorano, J., Gallego, J., Aragón-Salamanca, A., \& Gil de Paz, A. 2003, ApJ, 591, 827

Shapley, A. E., Steidel, C. C., Pettini, M., \& Adelberger, K. 2003, ApJ, 588, 65

Steidel, C. C., Adelberger, K. L., Giavalisco, M., Dickinson, M., \& Pettini, M. 1999, ApJ, 519, 1

Sullivan, M., Treyer, M. A., Ellis, R. S., Bridges, T. J., Milliard, B., \& Donas, J. 2000, MNRAS, 312, 442

Tresse, L., \& Maddox, S. J. 1998, ApJ, 495, 691

Tresse, L., \& Maddox, S. J., Le Fèvre, O., \& Cuby, J.-G. 2002, MNRAS, 337, 369

Treyer, M. A., et al. 2005, ApJ, 619, L19

Wang, B., \& Heckman, T. M. 1996, ApJ, 457, 645

Wilson, G., Cowie, L. L., Barger, A. J., \& Burke, D. J. 2002, AJ, 124, 1258

Wolf, C., Meisenheimer, K., Rix, H.-W., Borch, A., Dye, S., \& Kleinheinrich,

M. 2003, A\&A, 401, 73

Wyder, T. et al. 2005, ApJ, 619, L15 